\title{
Integrating Osteopathic Manipulative Treatment and Injections in the Diagnosis and Management of a Hip Labral Tear
}

Laura L. Snyder, OMS, IV; Shaun C. Knox, DO, PharmD; Charles J. Smutny, DO

From the Campbell University Jerry M. Wallace School of Osteopathic Medicine in Buies Creek, North Carolina. Financial Disclosures: None reported.

Support: None reported.

Address correspondence to Laura L. Snyder, OMS IV, 143 Main St, Buies Creek, NC 27506-0567.

Email: Ilstauber0802@email. campbell.edu

Submitted May 9, 2019; revision received August 4, 2019;

accepted August 25, 2019.
Hip pain in adults is a common complaint. Secondary processes can often mask underlying conditions, prolonging the course to diagnosis and definitive treatment. In this case, a 74-year-old man presented to an outpatient clinic after 6 weeks of right hip pain that began upon initiation of a rehabilitation program following coronary artery bypass graft surgery. Various imaging and treatment modalities (including radiography, osteopathic manipulative treatment, injection of the greater trochanteric bursa, L2-3 paravertebral block, magnetic resonance imaging, and intra-articular hip injection with $1 \%$ lidocaine) ultimately led to the definitive diagnosis of a labral tear. This case report highlights how a comprehensive osteopathic approach was used to evaluate and treat a patient with persistent unilateral hip pain because of an acetabular labral tear, associated psoas syndrome, and trochanteric bursitis, all within 2 months and with a positive clinical outcome.

J Am Osteopath Assoc. 2020;120(6):421-424

doi:10.7556/jaoa.2020.065

Keywords: hip pain, labral tear, osteopathic manipulative treatment

$\mathrm{P}$ hysicians often see patients with hip pain, yet because of the broad differential diagnoses, discovering a primary cause can be challenging. Secondary processes, such as inflammation, impaired biomechanics, and referred pain, can create confounding symptoms and mask underlying issues. Common causes include osteoarthritis, fracture, bursitis, labral tear, femoroacetabular impingement, referred lumbar or knee pain, and muscle spasm. ${ }^{1,2}$ Because of overlapping symptoms, acetabular labral tears often go undiagnosed for prolonged periods, with some studies suggesting a mean of more than 2 years before definitive diagnosis. This extended time to diagnosis can affect a patient's quality of life through limitations in physical function and persistent pain. ${ }^{3}$ Two studies ${ }^{4,5}$ have suggested that a labral lesion doubles the relative risk of significant chondral erosion and that the labrum has a low potential for self-repair because of a limited blood supply. With an estimated prevalence of $22 \%$ to $55 \%$ in patients with hip or groin pain, labral tears are a relevant concern for both primary care providers and orthopedic surgeons. ${ }^{3}$ This case report demonstrates how a comprehensive osteopathic approach was used to efficiently diagnose a labral tear in 2 months, as well as manage commonly associated sources of hip pain in an adult.

\section{Report of Case}

A 74-year-old man presented to our clinic with 6 weeks of right hip pain that began during a cardiac rehabilitation program. The patient had a significant medical history of 
coronary artery disease and degenerative disc disease; he had coronary artery bypass surgery 4 months prior to his initial clinic visit (Figure 1). The patient reported generalized pain in the right hip and low back that was most severe over the right greater trochanter. Positive physical examination findings included limited right hip extension, hypertonicity of the right iliopsoas muscle, and tenderness to palpation over the right greater trochanter. A decision was made to treat trochanteric bursitis and psoas syndrome using osteopathic manipulative treatment (OMT) and injection of the right trochanteric bursa using $4 \mathrm{~mL}$ of $1 \%$ lidocaine and $8 \mathrm{mg}$ of dexamethasone. Muscle energy and direct inhibition were used, with an emphasis on treating the psoas muscle. After treatment, there was an improvement in range of motion (ROM) of the hip, decreased hypertonicity of the psoas, and relief of pain. The patient was recommended home stretches and exercises focusing on the gluteus medius. He reported 3 to 5 days of symptomatic relief.

At follow-up, pain and restricted ROM had returned, and a right-on-right sacral torsion and L2 extended, rotated right, and sidebent right were present. Radiography of the right hip showed preserved joint space with minimal degeneration, suggesting a cause other than osteoarthritis or bony injury as the source of pain (Figure 2). The patient had pain in the right side of the groin that was reproducible with palpation over the right transverse processes of the L2-3 intertransverse space. A paravertebral block aimed at the L2-3 intertransverse space was performed using $4 \mathrm{~mL}$ of $1 \%$ lidocaine and $5 \mathrm{mg}$ of dexamethasone, resulting in pain reduction and postural improvement. Magnetic resonance imaging (MRI) of the right hip was ordered because of the recurrence of pain without evidence of bony involvement on plain radiographs; it showed appropriate joint space, no evident tendinopathy, and a possible anterosuperior labral tear. A diagnostic ultrasonography-guided intra-articular injection of the right hip was performed using $5 \mathrm{~mL}$ of $1 \%$ lidocaine and eliminated pain at the time of injection, further supporting a diagnosis of labral tear. After discussing treatment options, the patient decided on pursuing an intra-articular platelet-rich plasma injection if the pain returned. At a 6-month follow-up visit, the patient reported no return of significant pain and planned to wait for further treatment until necessary.

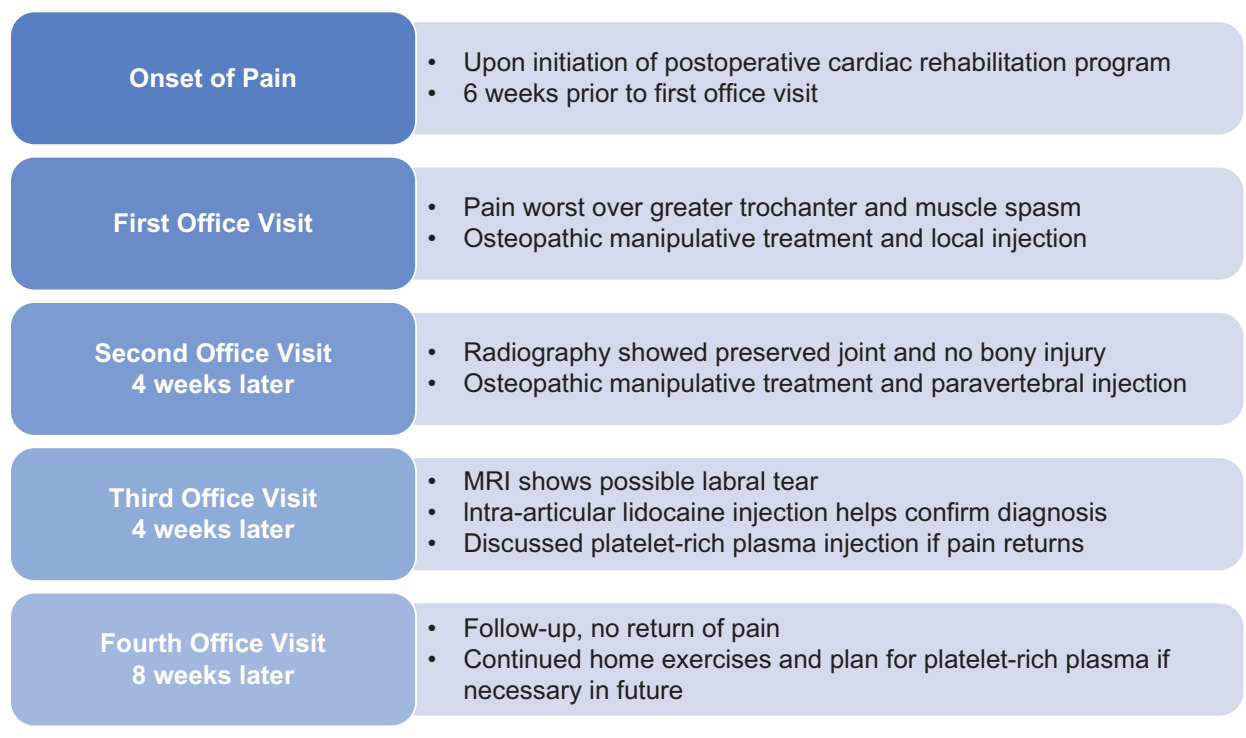

Figure 1.

Diagnosis and management of a patient reporting to an outpatient clinic with 6 weeks of right hip pain that began during a cardiac rehabilitation program following coronary artery bypass graft surgery. Abbreviation: MRI, magnetic resonance imaging. 


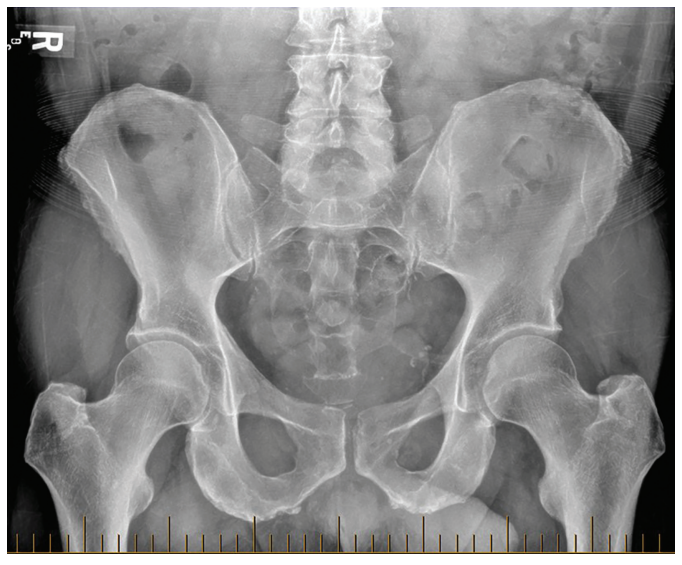

Figure 2.

Anterior-posterior pelvis view of a 74-year-old male demonstrating preserved joint space, minimal degenerative changes, and no acute fractures.

\section{Discussion}

The hip is a ball-and-socket joint comprising the articulation of the femoral head with the acetabulum, surrounded by a dense capsule. The acetabular labrum is a fibrocartilaginous structure that lines the acetabular socket; it is 2 to $3 \mathrm{~mm}$ thick and extends 2 to $3 \mathrm{~mm}$ past the acetabular rim. ${ }^{6}$ Vascular supply to the labrum comes from the obturator artery, superior gluteal artery, and inferior gluteal artery. ${ }^{5}$ Microvascular studies demonstrate that the labrum is avascular except for the superficial capsular surface, which suggests limitations in its innate healing potential. ${ }^{5}$ Patients with acetabular labral tears often present with anterior hip or groin pain, but half of patients may also complain of pain radiating to the lateral hip, anterior thigh, and buttocks. ${ }^{1,3}$ Labral tears can cause pain, changes in gait, instability of the hip, and restricted ROM, which may lead to secondary issues. ${ }^{2,3}$ Because of varying presentations, it is essential to have a comprehensive approach when evaluating hip pain for an accurate diagnosis.

Trochanteric bursitis is often the result of friction between the iliotibial band and the greater trochanter. The strain on the gluteus tendons attaching to the greater trochanter and alterations in gait and posture also contribute to localized inflammation. Initial treat- ment is usually conservative, focusing on nonsteroidal anti-inflammatory drugs, stretching, and physical therapy. Corticosteroid injection into the bursa is another common treatment modality. Surgical options, including iliotibial band release or bursal debridement, are available for pain duration of longer than 6 months. $^{7}$

Psoas syndrome is a musculoskeletal condition often overlooked by physicians. Because of the anatomic location of the psoas muscle, it can be affected by visceral or musculoskeletal structures. The psoas major muscle attaches to the transverse processes of T12-L5 vertebrae, intervening intervertebral discs, and the lesser trochanter of the femur. ${ }^{8}$ It is innervated by anterior rami of lumbar nerves L1-3 and contains the lumbar plexus embedded in the posterior aspect of the muscle. ${ }^{8}$ The psoas fascia has attachments to lumbar vertebrae, the pelvic brim, the quadratus lumborum fascia, and the thoracolumbar fascia, and it thickens superiorly to form the medial arcuate ligament. ${ }^{8}$ These connections demonstrate how dysfunction of the psoas can cause diffuse pain and disability, possibly complicating the diagnosis of lower back and hip pain.

OMT - including muscle energy, articulatory techniques, and myofascial release - has been shown to be an effective form of treatment for patients with psoas syndrome. $^{2}$ In the current case, similar OMT techniques were performed to manage psoas dysfunction, lumbar dysfunction, and sacral torsion, with resulting improvements in the patient's hip ROM, posture, and low back and hip pain. The innervation of the psoas muscle by L1-3 nerves explains associated somatic reflexes displayed throughout those vertebral segments. The patient had tenderness throughout the right transverse processes of L2-3 with referred pain to his groin. A paravertebral block was performed in the L2-3 interspace to desensitize the pathway and reduce inflammation. ${ }^{9}$ This technique provided symptomatic relief and postural improvement, suggesting that desensitization using local anesthetics can be a beneficial treatment option for patients with psoas syndrome. Because this patient had recurrent pain despite improvements in his 
psoas syndrome and trochanteric bursitis, further evaluation was necessary.

It has been suggested that hip physical examination findings do not provide significant value in the diagnosis of acetabular labral tears, further supporting the necessity of additional testing modalities. ${ }^{10}$ While both MRI and magnetic resonance angiography (MRA) have demonstrated efficacy in the diagnosis of labral tears, MRA has been suggested to be superior to MRI. ${ }^{3,11}$ In this case, MRA would have been a better test; however, labral tear was initially low on our differential diagnosis list because of secondary issues masking the true nature of the injury. Therefore, an ultrasonography-guided intra-articular injection of $1 \%$ lidocaine into the hip was performed and provided near-immediate pain relief, further solidifying the diagnosis of a labral tear. High sensitivity and specificity in the detection of intra-articular lesions using injections have been shown to have $90 \%$ accuracy of diagnostic hip injections when compared with arthroscopy. ${ }^{3}$

Early treatment for acetabular labral tears is often conservative using nonsteroidal anti-inflammatory drugs and physical therapy for at least 10 to 12 weeks; however, pain reoccurrence is common. Another option is intra-articular injection using local anesthetics with or without steroids. When conservative options fail, surgery can be successful, which also allows for direct visualization to assess for associated causes such as osteoarthritis. Hip arthroscopy is often used for labral debridement or repairs that can provide beneficial outcomes, including decreased pain, improved ROM, and decreased mechanical symptoms. ${ }^{3}$ In this case, the patient was not an appropriate surgical candidate because of his medical comorbidities; therefore, platelet-rich plasma injection was discussed as a possible treatment in the event his pain returned. Upon follow-up, the patient was pain-free and would continue home exercises and return for repeated injections, OMT, or platelet-rich plasma if necessary.

\section{Conclusion}

Labral tears of the hip generally have favorable outcomes with various treatment options once the diagnosis has been made. However, because of the variability in presentation and comorbid conditions, diagnosis can be complicated. Therefore, it is necessary to take a comprehensive approach when treating a patient with hip pain and low back pain. This case demonstrates how OMT and injections can be used along with traditional imaging methods to diagnose and manage an acetabular labral tear and associated conditions efficiently and effectively.

\section{References}

1. Wilson JJ, Furukawa M. Evaluation of the patient with hip pain. Am Fam Phys. 2014;89(1):27-34.

2. Tufo A, Desai GJ, Cox WJ. Psoas syndrome: a frequently missed diagnosis. J Am Osteopath Assoc. 2014;112(8):522-528. doi:10.7556/ jaoa.2012.112.8.522

3. Groh MM, Herrera J. A comprehensive review of hip labral tears. Curr Rev Musculoskelet Med. 2009;2(2):105-117. doi:10.1007/ s12178-009-9052-9

4. McCarthy JC, Noble PC, Schuck MR, Wright J, Lee J. The Otto E Aufranc Award: the role of labral lesions to development of early degenerative hip disease. Clin Orthop. 2001;393:25-37.

5. McCarthy JC, Noble PC, Schuck MR, WrightJ, Lee J. The watershed labral lesion: its relationship to early arthritis of the hip. J Arthroplast. 2001;16(8 suppl 1):81-87. doi:10.1054/arth.2001.28370

6. Bharam S. Labral tears, extra-articular injuries, and hip arthroscopy in the athlete. Clin Sports Med. 2006;25(2):279-292. doi:10.1016/j. csm.2006.01.003

7. Tibor LM, Sekiya JK. Differential diagnosis of pain around the hip joint. Arthroscopy. 2008;24(12):1407-1421. doi:10.1016/j.arthro.2008.06.019

8. Moore KL, Dalley AR, Agur AMR. Clinically Oriented Anatomy. 8th ed Wolters Kluwer; 2018

9. Lim H, Cho YH, Kim H, Lee DH, Kang SH. The effectiveness of L2 nerve root block for the management of patients who are suffering from chronic low back and referred pain. Korean J Anesthesiol. 2013;65(2):182-183. doi:10.4097/kjae.2013.65.2.182

10. Reiman MP, Goode AP, Cook CE, Holmich P, Thorborg K. Diagnostic accuracy of clinical tests for the diagnosis of hip femoroacetabular impingement/labral tear: a systematic review with meta-analysis. $\mathrm{Br}$ J Sports Med. 2014;49(12):811. doi:10.1136/bjsports-2014-094302

11. Smith TO, Hilton G, Toms AP, Donell ST, Hing CB. The diagnostic accuracy of acetabular labral tears using magnetic resonance imaging and magnetic resonance arthrography: a meta-analysis. Eur Radiol. 2011;21(4):863-874. doi:10.1007/s00330-010-1956-7

(c) 2020 American Osteopathic Association 